\title{
Tuberculin skin test and ELISPOT/T. SPOT.TB in children and adolescents with juvenile idiopathic arthritis
}

\author{
Flavio Sztajnbok ${ }^{1 *}$, Neio L F Boechat ${ }^{2}$, Samantha B Ribeiro ${ }^{3}$, Sheila K F Oliveira', Denise C N Sztajnbok ${ }^{4}$ \\ and Clemax C Sant'Anna ${ }^{5}$
}

\begin{abstract}
Background: There are controversies regarding the accuracy of the tuberculin skin test (TST) and methods based on the production of interferon gamma by sensitized T cells for the diagnosis of latent tuberculosis infection (LTBI) in pediatrics and immunosuppressed patients. Our objectives are to study TST and ELISPOT/T. SPOT.TB in the diagnosis of LTBI in children and adolescents with JIA undergoing methotrexate, the correlation between both and the sensitivity and specificity of T. SPOT.TB.

Methods: This is an observational prospective longitudinal study in which children and adolescents with JIA undergoing methotrexate therapy were assessed for clinical and epidemiological data for LTBI, in addition to performing TST and T. SPOT.TB at baseline and after 3 and 12 months.
\end{abstract}

Results: There were 24 patients. The prevalence of LTBI at inclusion was 20.8\%, the incidence after initiation of immunosuppressions $26.3 \%$ and the prevalence at the end of the study $41.6 \%$. Epidemiological history positive for TB showed a relative risk of 2.0 for the development of LTBI. Only 2 patients had positive T. SPOT.TB but only in one it was useful for detecting early LTBI. T. SPOT.TB presented a sensitivity of 10\%, specificity of $92.8 \%$, and low correlation with TST. No patient developed TB disease at a mean follow-up of 47 months.

Conclusions: We found a high prevalence of ILTB that doubled with immunosuppression and that epidemiological history was an important relative risk. T. SPOT.TB showed low sensitivity and high specificity, and no superiority over TST. There was low agreement and little influence of immunosuppression on the results of both tests.

\section{Background}

It is estimated that one third of the world's population is infected with Mycobacterium tuberculosis (M. tb) and $15-20 \%$ of new cases occur in children and adolescents [1]. In most cases, the infection remains latent. Nevertheless, latent tuberculosis infection (LTBI) can persist with risk of progression to disease in about $10 \%$ in the general population and $15-43 \%$ in the pediatric age group and is an important cause of morbidity and mortality, especially in immunossupressed patients $[2,3]$. LTBI is the clinical syndrome caused by exposure to $M$. $t b$ followed by infection, evidenced by the presence of positive tuberculin skin test (TST) in the absence of

\footnotetext{
* Correspondence: flaviosztajnbok@hotmail.com

${ }^{1}$ Pediatric Rheumatology Division, Universidade Federal do Rio de Janeiro, Rua Bruno Lobo 50, Cidade Universitária, 21941-912 Rio de Janeiro, Brasil Full list of author information is available at the end of the article
}

clinical and radiological signs and symptoms of disease activity $[4,5]$. To confirm the diagnosis of LTBI, epidemiological data, personal and familiar history, physical examination and laboratorial results should be considered. There is no confirmatory test for the diagnosis of LTBI and TST is still considered the gold standard, despite some limitations. In immunosuppressed patients, TST may present lower sensitivity and false negative results may occur, making LTBI diagnosis even more difficult [6].

Diagnostic tests in vitro using whole blood to assess the production of interferon-gamma (IFN - $\gamma$ ) by previously sensitized lymphocytes were developed in order to assist the diagnosis of tuberculosis (TB). These tests, known as IGRA (interferon-gamma release assay), are commercially available and the most frequently used are the QuantiFERON-TB In-Tube (enzyme linked immunosorbent assay) and T. SPOT.TB \%/ELISPOT (enzyme 
linked immunospot assay). They require just one visit and are not performed directly on the patient, reducing the possibility of occurring adverse effects, booster effect when repeated and abscence of the patient at the time of reading. However, they are expensive and require specialized laboratories.

In general, different studies have shown $60-80 \%$ agreement comparing TST and IGRAs in adults [7]. Some authors $[8,9]$ suggested that T. SPOT.TB presents better sensitivity and specificity than TST in children. But few studies have been conducted in immunosuppressed patients. The T. SPOT.TB assay has shown superiority compared to TST sensitivity and specificity for diagnosis of active TB in immunosuppressed patients [10]. However, most studies have been conducted in countries with low TB incidence and in adult population $[7,8]$. Studies to determine the sensitivity and specificity of IGRAs in immunocompromised children, especially from endemic areas, are still lacking.

Juvenile idiopathic arthritis (JIA) is the most common cause of chronic arthritis in pediatric age and its pathogenesis is associated with changes in the immune system [11]. This may explain the great predisposition of JIA patients to infections, beyond the fact that they frequently use immunosuppressive drugs as treatment [12]. In adults, rheumatoid arthritis itself, regardless the type of treatment, may be associated with increased incidence of $\mathrm{TB}$, and it raises with the use of immunosuppressive drugs, especially the modern biologic agents, but no data is known for children [13].

BCG is the only available vaccine for TB and provides protection against meningitis and disseminated forms. The World Health Organization (WHO) recommends that children from high-burden countries should be vaccinated at birth, a practice common in most of these countries. In low-burden countries this practice is restricted to children from high-risk groups for TB [4].

Early diagnosis and treatment of LTBI are challenges to be achieved in order to control TB worldwide. There is a need for more studies on the accuracy of diagnostic methods for TB in children and immunosuppressed patients, especially in high-burden countries. This study aimed to evaluate the frequency of LTBI and the sensitivity and specificity of the T. SPOT.TB compared to TST in the diagnosis of LTBI in patients with JIA before and after the use of methotrexate (MTX), as it is the first-choice secondline agent most frequently used in JIA [14], in a highburden country where most children receive BCG at birth.

\section{Methods}

This was an observational prospective longitudinal oneyear duration study approved by the local IRB. Children and adolescents with JIA (Edmonton Revision, 2001 [15]) and indication to use MTX without previous or current diagnosis of TB disease and no prior use of immunosuppressive drugs in the previous six months, were consecutively included from March 2008 to September 2011. All patients received MTX at a dosage of 10$15 \mathrm{mg} / \mathrm{m}^{2} /$ week as the first second line drug after nonsteroidal antiinflammatory drug (NSAID) failure, as the usual practice in our unit. If during the one-year followup corticosteroid or a biologic agent was necessary, the patient could continue in the study. However patients with indication to start a biologic agent were not included as these drugs are known to increase TB incidence [13] and they were already using MTX.

All patients answered a questionnaire to assess epidemiological data regarding $\mathrm{TB}$ at the first visit (T0). The intention was to evaluate contact with suspected cases of TB, presence of signs and symptoms suggestive of TB in patients and their families and previous treatment of LTBI or active disease. The questionnaire included previous BCG vaccination and the presence of the charactheristic skin scar (later confirmed by a physician), results of previous TST eventually performed, previous history, treatment or signs and symptoms of $\mathrm{TB}$ (patient and the family: unexplainned prolonged fever, prolonged cough, pulmonary or pleural disorders), socioeconomic conditions of the residence and neighborhood and contact with persons with TB.

At T0 chest radiography, TST, and basic laboratory tests such as complete blood count, acute phase reactants, assessment of renal and hepatic function, urinalysis, HIV and the T. SPOT.TB test were ordered. TST and T. SPOT.TB were also performed in the $3^{\text {rd }}(\mathrm{T} 3)$ and $12^{\text {th }}$ (T12) months. Chest radiography was repeated if either of the TB related tests became positive.

TST consisted of intradermal injection of $0.1 \mathrm{ml}$ of purified protein derivative (PPD RT $232 \mathrm{IU}$ ) of $M$. $t b$, according to local practice. Patients were considered to have a positive TST if an induration $\geq 5 \mathrm{~mm}$ diameter was present 48-72 hours after inoculation [4]. The $\mathrm{T}$. SPOT.TB/ELISPOT test was performed according to the manufacturer's recommendations. The test included sections with negative control (C-: no mitogens or antigens), sections with positive control ( $\mathrm{C}+$ : phytohemagglutinin) and sections to test two antigens (ESAT-6 and CFP-10). Spot-forming units (SFUs) were counted with the aid of a magnifying glass. Interpretation of the results was made as follows: when the negative control contained $\leq 5$ SFUs, this value was subtracted from the number of SFUs found in the section containing the antigens ESAT- 6 and CFP10 , and so: positive test $\geq 6$ SFUs for either antigen; negative test $\leq 6$ SFUs for both antigens and $\geq 20$ SFUs in the positive control section; indeterminate test $\leq 6$ SFUs for both antigens and $\leq 20$ SFUs in the positive control section. In case the negative control section presented with $\geq$ 6 SFUs, the test was considered positive if the number of 
SFUs in either antigen was greater than twice the number found in the negative control section. Samples of children with bacteriological TB were used as external control. The techinican performing the tests had no knowledge of clinical or laboratory data of the patients.

Descriptive statistics were used to obtain absolute frequencies and percentages for qualitative data, as well as means, medians, and standard deviations for quantitative data. The relationship between epidemiological history and the presence of LTBI was evaluated by chi-square test (significant if $\mathrm{p}$ value was less than 0.05 ). The sensitivity, specificity, positive predictive value (PPV) and negative predictive value (NPV) were calculated from $2 \times 2$ contingency tables, using the free program "Openepi". The kappa statistic was adopted to quantify the agreement between the results of TST and T. SPOT.TB using "Statistical Package for Social Sciences" (SPSS) version 16.0. The agreement was considered poor for kappa values less than 0.41 , moderate between 0.41 and 0.60 , substantial between 0.61 and 0.80 , and excellent when kappa was more than 0.81 .

\section{Results}

The study included 29 patients with JIA but because of non-adherence to the protocol before visit T3, five patients were discontinued and, thus, 24 patients remained in the study. Epidemiological and clinical data of the patients at baseline are shown in Table 1 . There were 17 female patients $(70.8 \%)$. Ten patients (41.7\%) were polyarticular rheumatoid-factor negative, nine (37.5\%) oligoarticular persistent and five (20.8\%) systemic. The mean time for MTX onset was 30 months after diagnosis and 35 months after disease onset. All children received BCG vaccine at birth as a current practice in Brazil. Twenty patients were using NSAIDs and four were out of medications at the time of MTX onset. During the one-year follow-up, no patient needed to use corticosteroid or a biologic agent.

At T0, eight patients had positive epidemiological history for TB and five of them developed LTBI. Of 16 patients without a positive epidemiological history, five developed LTBI. The relative risk was 2 for patients with epidemiological history for TB compared to those without. None of the patients with positive results for $\mathrm{T}$. SPOT.TB had a positive epidemiological history for TB. All 24 patients in the study had normal chest radiography at T0 and there were no reports of new positive epidemiological history of TB at T3 and T12.

At T0, before MTX onset, five patients already had a positive TST. At T12, no patient had developed TB disease and 10 had developed LTBI. Thus, LTBI was diagnosed in 5/24 (20.8\%) patients at T0 and 5/19 (26.3\%) patients after immunosuppression onset, what means 10/24 (41.6\%) patients from T0 to T12. All patients with positive TST were treated for LTBI with isoniazid for six months, and there was no occurrence of toxicity in any of them. All those with conversion of TST had a new chest X-ray, which was normal in all cases. No patient had a TST in the previous 2years before T0.

The agreement between TST and T. SPOT.TB could not be assessed at $\mathrm{T} 0$ due to the fact that all results were negative for the T. SPOT.TB. At T3, two patients had

Table 1 Epidemiological and clinical data from patients with JIA ( $\mathbf{n}=\mathbf{2 4}$ )

\begin{tabular}{|c|c|c|c|}
\hline Variables & & $\mathbf{n}$ & $\%$ \\
\hline \multirow[t]{2}{*}{ Gender } & Female & 17 & 70,8 \\
\hline & Male & 7 & 29,2 \\
\hline \multirow[t]{3}{*}{ JIA subtypes } & Polyarticular rheumatoid factor negative & 10 & 41,7 \\
\hline & Oligoarticular persistent & 9 & 37,5 \\
\hline & Systemic & 5 & 20,8 \\
\hline \multirow[t]{2}{*}{ Patients' age at JIA diagnosis (range; mean; median) } & $20-216$ & & \\
\hline & $(100 \pm 50 ; 92)$ & & \\
\hline \multirow[t]{2}{*}{ Timeframe between JIA diagnosis and inclusion in the study (range; mean; median) } & $1-108$ & & \\
\hline & $(30 \pm 38 ; 9)$ & & \\
\hline \multirow{2}{*}{$\begin{array}{l}\text { Disease duration: timeframe between JIA onset and inclusion in the study } \\
\text { (range; mean; median) }\end{array}$} & $2-108$ & & \\
\hline & $(35 \pm 37 ; 23)$ & & \\
\hline \multirow[t]{2}{*}{ Patients' age at inclusion (range; mean; median) } & $36-217$ & & \\
\hline & $(130 \pm 51 ; 119)$ & & \\
\hline \multirow[t]{2}{*}{ Epidemiological history of contact with TB at inclusion (T0) } & yes & 8 & 33,3 \\
\hline & no & 16 & 66,6 \\
\hline
\end{tabular}


positive T. SPOT.TB tests and, at T12, one of them had a negative test. Thus, there was a poor correlation between the two tools ( $\mathrm{K}=0.11$ and 0.16 , respectively).

To evaluate the sensitivity and specificity of the $T$. SPOT.TB, we considered TST value $\geq 5 \mathrm{~mm}$ as the gold standard. At T3 the sensitivity of T. SPOT.TB was $20 \%$ (CI 95\% $=3.6$ to 62.4 ) and the specificity $89.4 \%$ (CI 95\% = 68.6 to 97 ), and at T12 $12.5 \%$ (CI 95\% = 2.2 to 47 ) and $100 \%$ (CI $95 \%=80.6$ to 100 ), respectively. Taking into account that 10 patients had LTBI, the final sensitivity of the T. SPOT.TB was $10 \%$ (CI $95 \%=1.7$ to 40.4 ) and specificity was $92.8 \%$ (CI $95 \%=68.5$ to 98.7$)$. In this scenario, the PPV of the T. SPOT.TB was $50 \%$ (CI 95\% $=9.4$ to 90.5 ) and the NPV was $59 \%$ (CI $95 \%=38.7$ to 76.7 ). A ROC curve for T. SPOT.TB showed an area under the curve of 0.51 ( $\mathrm{p}$ value 0.81 ).

No other immunosuppressive therapy was initiated and no MTX discontinued during the one-year study follow-up. In the last appointment in March 2013, after a mean follow-up of 3 years and 11 months, no patient in the study had developed active TB. Table 2 shows clinical and epidemiological data besides TST and T. SPOT.TB results for each subject in the study.

\section{Discussion}

To our knowledge, this study was the first to assess the performance of TST and T. SPOT.TB in pediatric patients with a rheumatic disease in a high-burden country. Brazil presents a high incidence of $\mathrm{TB}$, around 38 cases per 100.000 inhabitants in 2011 [16].

In our study, the epidemiological history for TB doubled the relative risk for the development of LTBI compared to patients with negative epidemiological history. In a systematic review in which $40 \%$ of the studies were conducted in countries where TB was endemic, it was found an association between positive epidemiological history and responses in TST and IGRAs [17]. The authors suggested that an important marker to be considered for the diagnosis of LTBI would be the degree of exposure of the patient, i.e. location, number of hours of exposure to a person with TB and bacillary load. Unfortunately, in our study, such an assessment was virtually impossible because the lack of information provided by the assisted population. Thus, our study considered only yes/no responses concerning exposure.

The conversion of TST usually occurs about two to 12 weeks after infection and the T. SPOT.TB between four and 22 weeks [18]. In one patient (\#13), T. SPOT. TB presented a positive result prior to TST conversion and, at least in this case, T. SPOT.TB was sensitive enough to identify LTBI in advance. In the other patient (\#12), T. SPOT.TB became negative at T12 and TST remained negative. One explanation for this latter patient could be the occurrence of a booster effect because
IGRA in T3 was performed sometime after TST in this patient. This is a rare but possible event $[19,20]$.

No patient developed active TB during the one-year follow-up of the study. All patients had a prolonged follow-up after this initial proposed study time (one year), with a mean time of 3 years and 11 months, and although in this phase there were patients who started biologic agents, none of them developed active TB. We believe that this fact was possibly due to the early treatment of LTBI [21].

We did not find national or international data to compare our high rates of LTBI as most of the data available refers to active TB. The most comparable to ours, as conditions of patients at risk for acquiring LTBI, were reported rates of LTBI in $61.5 \%$ of subjects in a prison hospital, $40 \%$ of shelter residents, and $20 \%$ of injection drug users [22]. Besides the high incidence of TB in Brazil, additional factors that could explain the high rates of LTBI in our study are the immunosuppression associated with JIA itself, patients' frequent exposure to health services and possible TB, and the use of MTX.

Our study showed poor correlation between the results of TST and T. SPOT.TB. The few available studies showed that such an agreement appears to be low in countries where $\mathrm{TB}$ is endemic, unlikely in countries with low incidence, and this could be explained by the high sensitivity inherent to TST revealing false negative IGRAs $[5,19,23,24]$. It should be noted that patients received BCG at birth, many years before study participation, so BCG very probably did not influence TST results. It is known that TST positivity associated to BCG in the neonatal age decreases rapidly in a few years [25]. TST positivity could not be explained by a booster effect, as no patient had TST performed in the two years prior to the study onset.

Immunosuppression may affect the sensitivity of both TST and IGRAs [23,26]. Although in immunosuppressed adults IGRAs showed higher sensitivity than TST $[21,27,28]$, some authors found lower values for both TST and IGRAs in patients with rheumatoid arthritis using immunosuppressants compared to those not using immunosuppressants [23]. For the TST cut-off value of $5 \mathrm{~mm}$ as used in Brazil [4], IGRAs showed high specificity and low sensitivity. At this cut-off, TST performed better than T. SPOT.TB. In our study, it was difficult to evaluate the role of immunosuppression on the results of T. SPOT. $\mathrm{TB}$, since all were negative at T0, before MTX onset. Between T3 and T12 sensitivity, which was already low, slightly decreased. It was not possible to explain why four patients showed conversion of TST during the study and the T. SPOT.TB remained negative. Although TST and T. SPOT.TB evaluate different responses, it would be expected that immunosuppression could influence TST more often than IGRAs, since the former depends on the 
Table 2 Epidemiological and clinical data besides TST and T. SPOT.TB/ELISPOT results from the 24 cases

\begin{tabular}{|c|c|c|c|c|c|c|c|c|c|}
\hline Case/gender & JIA & $\begin{array}{l}\text { Age at diagnosis } \\
\text { (months) }\end{array}$ & $\begin{array}{l}\text { Time Dx-T0 } \\
\text { (months) }\end{array}$ & $\begin{array}{c}\text { Age T0 } \\
\text { (months) }\end{array}$ & TO & T3 & $\mathrm{T} 12$ & $\begin{array}{c}\text { Follow up } \\
\text { (months) }\end{array}$ & LTBI \\
\hline 1 & $P$ & 72 & 16 & 88 & TST 0 & TSTO & TST 0 & 57 & \\
\hline M & & & & & Elispot - & Elispot - & Elispot - & & \\
\hline $2^{*}$ & $\mathrm{O}$ & 20 & 90 & 110 & TST 0 & TST 4 & TST 10 & 58 & yes \\
\hline $\mathrm{F}$ & & & & & Elispot - & Elispot - & Elispot - & & \\
\hline 3 & $P$ & 152 & 2 & 154 & TST 7 & TST 0 & TST 0 & 59 & yes \\
\hline$F$ & & & & & Elispot - & Elispot - & Elispot - & & \\
\hline 4 & $\mathrm{O}$ & 192 & 24 & 216 & TST 0 & TST 0 & TST 0 & 59 & \\
\hline$F$ & & & & & Elispot - & Elispot - & Elispot - & & \\
\hline $5^{*}$ & $P$ & 130 & 6 & 136 & TST 5 & TST 19 & TST 12 & 55 & yes \\
\hline $\mathrm{F}$ & & & & & Elispot - & Elispot - & Elispot - & & \\
\hline $6^{*}$ & $\mathrm{O}$ & 88 & 1 & 89 & TST 0 & TST 0 & TST 0 & 56 & \\
\hline M & & & & & Elispot - & Elispot - & Elispot - & & \\
\hline $7^{*}$ & $\mathrm{~S}$ & 102 & 2 & 104 & TST 0 & TST 10 & TST 10 & 56 & Yes \\
\hline $\mathrm{F}$ & & & & & Elispot - & Elispot - & Elispot - & & \\
\hline 8 & O & 84 & 106 & 190 & TST 14 & TST 10 & TST 15 & 56 & Yes \\
\hline $\mathrm{F}$ & & & & & Elispot - & Elispot - & Elispot - & & \\
\hline 9 & O & 108 & 99 & 207 & TST 13 & TST 15 & TST 13 & 56 & Yes \\
\hline M & & & & & Elispot - & Elispot - & Elispot - & & \\
\hline 10 & O & 86 & 30 & 116 & TST 0 & TST 0 & TST 0 & 55 & \\
\hline$F$ & & & & & Elispot - & Elispot - & Elispot - & & \\
\hline 11 & $S$ & 113 & 1 & 114 & TST 0 & TST 0 & TST 0 & 53 & \\
\hline $\mathrm{F}$ & & & & & Elispot - & Elispot - & Elispot - & & \\
\hline 12 & $S$ & 24 & 46 & 70 & TST 0 & TST 0 & TST 0 & 37 & \\
\hline F & & & & & Elispot - & Elispot + & Elispot - & & \\
\hline 13 & P & 139 & 4 & 143 & TST 0 & TST 0 & TST 10 & 52 & Yes \\
\hline M & & & & & Elispot - & Elispot + & Elispot + & & \\
\hline $14^{*}$ & $P$ & 111 & 12 & 123 & TST 3 & TST 3 & TST 0 & 51 & \\
\hline M & & & & & Elispot - & Elispot - & Elispot - & & \\
\hline $15^{*}$ & O & 85 & 108 & 193 & TST 0 & TST 0 & TST 0 & 50 & \\
\hline $\mathrm{F}$ & & & & & Elispot - & Elispot - & Elispot - & & \\
\hline 16 & $S$ & 54 & 48 & 102 & TST 1 & TST 0 & TST 0 & 29 & \\
\hline $\mathrm{F}$ & & & & & Elispot - & Elispot - & Elispot - & & \\
\hline 17 & $\mathrm{O}$ & 60 & 90 & 150 & TST 0 & TST 0 & TST 0 & 29 & \\
\hline$F$ & & & & & Elispot - & Elispot - & Elispot - & & \\
\hline 18 & $S$ & 66 & 1 & 67 & TST 0 & TST 0 & TST 0 & 28 & \\
\hline$F$ & & & & & Elispot - & Elispot - & Elispot - & & \\
\hline $19^{*}$ & $P$ & 156 & 32 & 188 & TST 0 & TST 0 & TST 23 & 25 & Yes \\
\hline M & & & & & Elispot - & Elispot - & Elispot - & & \\
\hline 20 & $P$ & 160 & 1 & 161 & TST 0 & TST 0 & TST 0 & 19 & \\
\hline $\mathrm{F}$ & & & & & Elispot - & Elispot - & Elispot - & & \\
\hline 21 & $P$ & 61 & 1 & 62 & TST 0 & TST 0 & TST 0 & 18 & \\
\hline $\mathrm{F}$ & & & & & Elispot - & Elispot - & Elispot - & & \\
\hline 22 & O & 35 & 1 & 36 & TST 0 & TST 10 & TST 10 & 60 & Yes \\
\hline $\mathrm{F}$ & & & & & Elispot - & Elispot - & Elispot - & & \\
\hline
\end{tabular}


Table 2 Epidemiological and clinical data besides TST and T. SPOT.TB/ELISPOT results from the 24 cases (Continued)

\begin{tabular}{cccccccccc}
\hline 23 & $P$ & 96 & 98 & TST 0 & TST 0 & TST 0 & 57 \\
$F$ & & & & Elispot - & Elispot - & Elispot - \\
$24^{*}$ & $P$ & 216 & 1 & 217 & TST 5 & TST 4 & TST 2 & 57 \\
$M$ & & & & Elispot - & Elispot - & Elispot - & \\
\hline
\end{tabular}

Legend:

- JIA = JIA subtypes: $O$ = oligoarticular $J \mathrm{~A} ; \mathrm{P}=$ polyarticular $\mathrm{JI} ; \mathrm{S}=$ systemic $\mathrm{J} \mathrm{A}$.

$\cdot(*)=$ epidemiological hystory positive for TB.

- TST: in millimeters.

- T.SPOT.TB/ELISPOT : $(-)=$ negative; $(+)=$ positive.

- T0: study onset; T3: 3rd month; T12: 12th month.

individual hypersensitive and T-cell proliferation [25,29]. Therefore, in our patients, immunosuppression from MTX did not influence the results of T. SPOT.TB.

T. SPOT.TB in children from endemic areas has shown lower sensitivity and higher specificity than TST $[30,31]$. It had been shown that IGRAs presented better accuracy than the TST for the diagnosis of TB in immunocompromised children [17], but a recent study showed that IGRAs in children and immunosuppressed patients might present false-negative results in endemic areas for TB [32]. Throughout our study, T. SPOT.TB showed low sensitivity but high specificity. If a positive T. SPOT.TB was used for the diagnosis and indication for treatment of LTBI in our patients, only two of them would have been diagnosed as LTBI and treated. This could mean that, in a high-burden setting, patients might be untreated. On the other hand, it has been proposed that in low-burden settings replacing TST with IGRA for determining LTBI could allow a reduction in the number of patients receiving treatment [28].

A study with epidemiological characteristics similar to ours compared the use of TST and T. SPOT.TB in the diagnosis of LTBI in adult patients with rheumatoid arthritis and indication for use of biological agents. The specificity of the T. SPOT.TB ranged from $87 \%$ to $90 \%$ and NPV from $94.4 \%$ to $100 \%$, with similar sensitivity to TST. Authors suggested that the low sensitivity of T. SPOT.TB in endemic areas for TB could be explained by the fact that the antigens in the T. SPOT.TB plate, although specific for $M . t b$, represented a small portion of numerous antigens, and more reliable results could be achieved by adding new antigens to the method [33]. In Gambia, a TB- endemic region, T. SPOT.TB and TST had similar results in the diagnosis of LTBI in children vaccinated with BCG at an early age. TST and IGRA in conjunction increased sensitivity in only $10 \%$ [20].

Our study had limitations, but many of them bring us to real-life conditions, especially in developing countries. The low prevalence of JIA might explain the difficulty to include patients [11] and, unfortunatelly, there was no possibility to test the real state of immunosuppression in patients by assessing CD4 and CD8 counts.
Recently, WHO recommended that IGRAs should not be used in low- and middle-income countries (generally those with higher TB burden), as there are insufficient data and evidence on the performance of IGRAs in these populations [4]. IGRAs have similar performance to TST but are more expensive and complex to perform $[4,34]$ and, therefore, TST should be preferred in these settings. However, data from pediatric population and immunosuppressed individuals who could benefit from IGRAs are limited and more studies with large sample sizes are desirable.

\section{Conclusion}

In conclusion, we found a high frequency of LTBI among patients with JIA, which doubled after one year of MTX therapy. There was a low agreement between TST and T. SPOT.TB, low sensitivity and high specificity for the T. SPOT.TB, and small influence of immunosuppression related to MTX use on the performance of both. Even with MTX, no cases treated for LTBI developed active TB. There was no superiority of T. SPOT.TB compared to TST for the diagnosis and monitoring of LTBI in childen with JIA using MTX.

Competing interest

The authors declare that they have no competing interest.

\section{Authors' contribution}

FS and all co-authors participated in the conception, design, analysis and interpretation of data. SBR also performed all Elispot tests. All authors read and approved the final manuscript.

\section{Acknowledgements}

We thank Drs. Marta Christine F. Rodrigues, Christianne Diniz, Maria Vitoria H. Seidl, Eduardo Sica Pinheiro, Gustavo Guimarães, Leandro Vieira and Carine Sobreira for collaborating in the study.

\section{Author details}

${ }^{1}$ Pediatric Rheumatology Division, Universidade Federal do Rio de Janeiro, Rua Bruno Lobo 50, Cidade Universitária, 21941-912 Rio de Janeiro, Brasil. ${ }^{2}$ Pneumology Division, Universidade Federal do Rio de Janeiro, Rua Rodolpho Paulo Rocco 255, Cidade Universitária, 21941-913 Rio de Janeiro, Brasil. ${ }^{3}$ Molecular Biology Division, Multidisciplinary Laboratory, Hospital Universitário Clementino Fraga Filho, Universidade Federal do Rio de Janeiro, Rua Rodolpho Paulo Rocco 255, Cidade Universitária, 21941-913 Rio de Janeiro, Brasil. ${ }^{4}$ Pediatric Infectious Diseases Division, Universidade do Estado do Rio de Janeiro, Avenida 28 de setembro 87, Vila Isabel, 20551-030 Rio de Janeiro, Brasil. ${ }^{5}$ Pediatric Pneumology Division, Universidade Federal do Rio 
de Janeiro, Rua Bruno Lobo 50, Cidade Universitária, 21941-912 Rio de Janeiro, Brasil.

Received: 24 January 2014 Accepted: 12 May 2014

Published: 22 May 2014

\section{References}

1. World Health Organization: Global tuberculosis control: surveillance, planning financing. Geneva, Switzerland: World Health Organization; 2011

2. Dye C, Scheele S, Dolin P, Pathania V, Raviglione MC: Consensus statement. Global burden of tuberculosis: estimated incidence, prevalence and mortality by country. WHO Global Surveillance and monitoring project. JAMA 1999, 282:6776-6786.

3. Shingadia D, Novelli V: Diagnosis and treatment of tuberculosis in children. Lancet Infect Dis 2003, 3:624-32.

4. World Health Organization: Guidance for national tuberculosis programmes on the management of tuberculosis in children. 2nd edition. Geneva, Switzerland: World Health Organization; 2014.

5. Connell TG, Ritz N, Paxtomn GA, Buttery JP, Curtis N, Ranganathan SC: Three-way comparison of tuberculin skin testing, QuantiFERON-TB GOLD and T-SPOT.TB in children. PLoS One 2008, 3:e2624.

6. Pai M, Lewinsohn DM: Interferon- $\gamma$ assays for tuberculosis. Is anergy the Achilles' heel? Am J Resp. Crit Care Med 2005, 172:519-521.

7. Dheda K, van Zyl Smit R, Badri M, Pai M: T-cell interferon-gamma release assays for the rapid immunodiagnosis of tuberculosis: clinical utility in high-burden vs. low-burden settings. Curr Opin Pulm Med 2009, 15:188-200

8. Richeldi L, Ewer K, Losi M, Bergamini BM, Roversi P, Deeks J, Fabbri LM, Lalvani A: T cell-based tracking of multidrug resistant tuberculosis infection after brief exposure. Am J Respir Crit Care Med 2004 170:288-295.

9. Soysal A, Millington KA, Bakir M, Dosanjh D, Aslan Y, Deeks JJ, Efe S, Staveley I, Ewer K, Lalvani A: Effect of BCG vaccination on risk of Mycobacterium tuberculosis infection in children with household tuberculosis contact: a prospective community-based study. Lancet 2005, 366:1443-1451.

10. Murakami S, Takeno M, Kirino Y: Detection of Mycobacterium tuberculosis antigen specific immune responses by using ELISPOT technique in rheumatoid artritis patients receiving immunosuppressive therapies. Arthritis Rheum 2006, 9(suppl):S517.

11. Andersson GB: Juvenile arthritis: Who gets it, where and when? A review of current data on incidence and prevalence. Clin Exp Rheumatol 1999, 17:367-374

12. Woo P: Cytokines and juvenile idiopathic arthritis. Curr Rheumatol Rep 2002, 4:452-457.

13. Baronnet L, Barnetche T, Kahn V, Lacoin C, Richez C, Schaeverbeke T: Incidence of tuberculosis in patients with rheumatoid arthritis: A systematic literature review. Joint Bone Spine 2011, 78:279-284.

14. Singsen $\mathrm{BH}$, Goldbach-Mansky R: Methotrexate in the treatment of juvenile rheumatoid arthritis and other pediatric rheumatoid and nonrheumatic disorders. Rheum Dis Clin North Am 1997, 23(4):811-840.

15. Petty RE, Southwood TR, Manners P, Baum J, Glass DN, Goldenberg J, He X, Maldonado-Cocco J, Orozco-Alcala J, Prieur AM, Suarez-Almazor ME, Woo P, International League of Associations for Rheumatology: International League of Associations for Rheumatology classification of juvenile idiopathic arthritis: second revision, Edmonton, 2001. J Rheumatol 2004, 31:390-392.

16. Brazilian Ministry of Health. [http://portalsaude.saude.gov.br/portalsaude]

17. Mandalakas AM, Detjen AK, Hesseling AC, Benedetti A, Menzies D: Interferon-gamma release assays and childhood tuberculosis: systematic review and meta-analysis. Int J Tuberc Lung Dis 2011, 15:1018-1032.

18. Lee SW, Oh DK, Lee SH, Kang HY, Lee C-T, Yim J-J: Time interval to conversion of interferon- $\gamma$ release assay after exposure to tuberculosis. Eur Respir J 2011, 37:1447-1452.

19. Cruz AT, Geltemeyer AM, Starke JR, Flores JA, Graviss EA, Smith KC Comparing the tuberculin skin test and T-SPOT.TB blood test in children. Pediatrics 2011, 127:e31-38.

20. Adetifa IMO, Ota MOC, Jeffries DJ, Hammond A, Lugos MD, Donkor S, Patrick O, Adegbola RA, Hill PC: Commercial interferon gamma release assays compared to the tuberculin skin test for the diagnosis of latent Mycobacterium tuberculosis in childhood contacts in the Gambia. Pediatr Infect Dis J 2010, 29:439-443.
21. Keane J, Bresnihan B: Tuberculosis reactivation during immunosupressive therapy in rheumatic diseases: diagnostic and therapeutic strategies. Curr Opin Rheumatol 2008, 20:443-449.

22. Lemos ACM, Matos ED, Bittencourt CN: Prevalência de TB ativa e TB latente em internos de um hospital penal na Bahia. J Bras Pneumol 2009, 35:63-68.

23. Hanta I, Ozbek S, Kuleci S, Seydaoglu G, Ozyyilmaz E: Detection of latent tuberculosis infection in rheumatologic diseases before anti-TNFa therapy: tuberculin skin test versus IFN- $\gamma$ assay. Rheumatol Int 2012, 32:3599-3603.

24. Machado A Jr, Emodi K, Takenami I, Finkmoore BC, Barbosa T, Carvalho J, Cavalcanti L, Santos G, Tavares M, Mota M, Barreto F, Reis MG, Arruda S, Riley LW: Analysis of discordance between the tuberculin skin test and the interferon-gamma release assay. Int J Tuberc Lung Dis 2009, 13:446-453.

25. Farhat M, Greenaway C, Pai M, Menzies D: False-positive tuberculin skin tests: what is the absolute effect of BCG and non-tuberculous mycobacteria? Int J Tuberc Lung Dis 2006, 10:1192-1204.

26. Perez-Velez CM, Marais BJ: Tuberculosis in children. N Engl J Med 2012, 367:348-361.

27. Matulis G, Jüni $P$, Villiger PM, Gadola SD: Detection of latent tuberculosis in immunosuppressed patients with autoimmune diseases: performance of a Mycobacterium tuberculosis antigen-specific interferon-gamma assay. Ann Rheum Dis 2008, 67:84-90.

28. Mariette X, Baron G, Tubach F, Lioté F, Combe B, Miceli-Richard C, Flipo R-M, Goupille P, Allez M, Salmon D, Emilie D, Carcelain G, Ravaud P: Influence of replacing tuberculin skin test with ex vivo interferon $\gamma$ release assays on decision to administer prophylactic antituberculosis antibiotics before anti-TNF therapy. Ann Rheum Dis 2012, 71:1783-1790.

29. Pabst HF, Kreth HW: Ontogeny of the immune response as a basis of childhood diseases. J Pediatr 1980, 97:519-534.

30. Lalvani A, Millington KA: T cell-based diagnosis of childhood tuberculosis infection. Curr Opin Infect Dis 2007, 20:264-271.

31. Molicotti P, Bua A, Mela G, Olmeo P, Delogu R, Ortu S, Sechi LA, Zanetti S: Performance of QuantiFERON-TB testing in a tuberculosis outbreak at a primary school. J Pediatr 2008, 152:585-586.

32. Abubakar I, Stagg HR, Whitworth $H$, Lalvani A: How should I interpret gamma release assay result for tuberculosis infection? Thorax 2013, 68:298-301.

33. Marques CD, Duarte AL, de Lorena VM, Souza JR, Souza WV, Gomes YM, de Carvalho EM: Evaluation of an interferon gamma assay in the diagnosis of latent tuberculosis infection in patients with rheumatoid arthritis. Rheumatol Int 2009, 30(1):57-62.

34. Rangaka MX, Wilkinson KA, Glynn JR, Ling D, Menzies D, MwansaKambafwile J, Fielding K, Wilkinson RJ, Pai M: Predicitive value of interferon-gamma release assays for incident active tuberculosis: a systematic review and meta-analysis. Lancet Infect Dis 2012, 12:45-55.

doi:10.1186/1546-0096-12-17

Cite this article as: Sztainbok et al. Tuberculin skin test and ELISPOT/T. SPOT.TB in children and adolescents with juvenile idiopathic arthritis. Pediatric Rheumatology 2014 12:17.

\section{Submit your next manuscript to BioMed Central and take full advantage of:}

- Convenient online submission

- Thorough peer review

- No space constraints or color figure charges

- Immediate publication on acceptance

- Inclusion in PubMed, CAS, Scopus and Google Scholar

- Research which is freely available for redistribution 